\title{
Implementation of Four Real-Time Software Defined Receivers and a Space-Time Decoder using Xilinx Virtex 2 Pro Field Programmable Gate Array
}

\author{
Peter J. Green and Desmond P. Taylor \\ Department of Electrical and Computer Engineering University of Canterbury \\ Christchurch \\ New Zealand \\ peter.green@canterbury.ac.nz \\ taylor@elec.canterbury.ac.nz
}

\begin{abstract}
This paper describes the concept, architecture, development and demonstration of a real time, high performance, software defined 4-receiver system and a space time decoder to be implemented on a Xilinx Virtex 2 Pro Field Programmable Gate Array. It is designed and developed for research into receiver diversity and multiple input and multiple output (MIMO)wireless systems. Each receiver has a Freescale DSP56321 digital signal processor (DSP) to run synchronization, channel state estimation and equalization algorithms. The system is software defined to allow for flexibility in the choice of receiver demodulation formats, output data rates and space-time decoding schemes. Hardware, firmware and software aspects of the receiver and space time decoder system to meet design requirements are discussed.
\end{abstract}

The current system implementation is an enhancement to an existing Smart Antenna Software RAdio Test System (SASRATS) platform [5, 6] designed to test and verify various space time architectures and algorithms. Our original receivers, developed in 2000 [5] were designed for low bandwidth (270 KBauds) and beamforning/smart antenna work. The latest enhancement allows high bandwidth performance (4 MBauds), parallel 16-bit IQ baseband data outputs and greater programmability. The 4 receivers complement a 4 transmitter space time (ST) encoding platform designed and developed for real-time testing of ST coding schemes developed by Alamouti [1] and others mentioned in [4]. The primary objective is to increase system capacity and performance through the use of multiple antennas, employing spatial multiplexing and ST coding and decoding.

The focus of this paper is on the digital and baseband portion of the system. The analogue portion consists of an RF amplifier, a phase locked local oscillator, a mixer and in- termediate frequency (IF) bandpass filters and high gain IF amplifiers whose purpose is to amplify, translate and filter a received radio frequency signal at $915 \mathrm{MHz}$ or $2.4 \mathrm{GHz}$ to an intermediate frequency (IF) of $70 \mathrm{MHz}$ where digitization and bandpass sampling occurs. The architecture of the digital IF and baseband portion of the receivers is shown in Figure 1.

The 70 Mhz IF signal is digitised using bandpass sampling techniques using a high speed Analog Devices analogto-digital convertor (ADC) AD6640 at a sampling clock frequency of $65 \mathrm{MHz}$. The sampling clock frequency is fully programmable and is generated from a Direct Digital Synthesizer (DDS) from a AD9857 Upconvertor integrated circuit (IC) working in fixed tone mode. This allows us the flexibility to change the sampling clock frequency for various experiments. The output of the ADC is then fed into a versatile Analog Devices AD6620 Digital Down Converter (DDC) which digitally downconverts, decimates and filters the input data to baseband IQ signals for further processing. The AD6620 is fully programmable and together with the AD9857, makes the receiver software defined. However, one is restricted by the chip design to resolution of the output data words to 16-bits and the sequential parallel 16-bit output format of the in-phase (I) and quadrature phase $(\mathrm{Q})$ signals. The 16-bit sequential I and $\mathrm{Q}$ data are stored in 2 sets of 32-bit buffers. A Data_Valid pulse is generated after the IQ data are latched into the buffers. The IQ data can then be accessed as a simultaneous parallel 32bit output pair or as separate I and Q data words, using memory mapped addressing under DSP or FPGA control. Simultaneous 32 bit parallel transfer is normally used between the DDC and a National Instruments Data Acquisition (NIDAQ) card which is used with the SASRATS platform to perform batch or pseudo real-time processing of data using MATLAB. This feature is useful during development and testing stages of the design. 


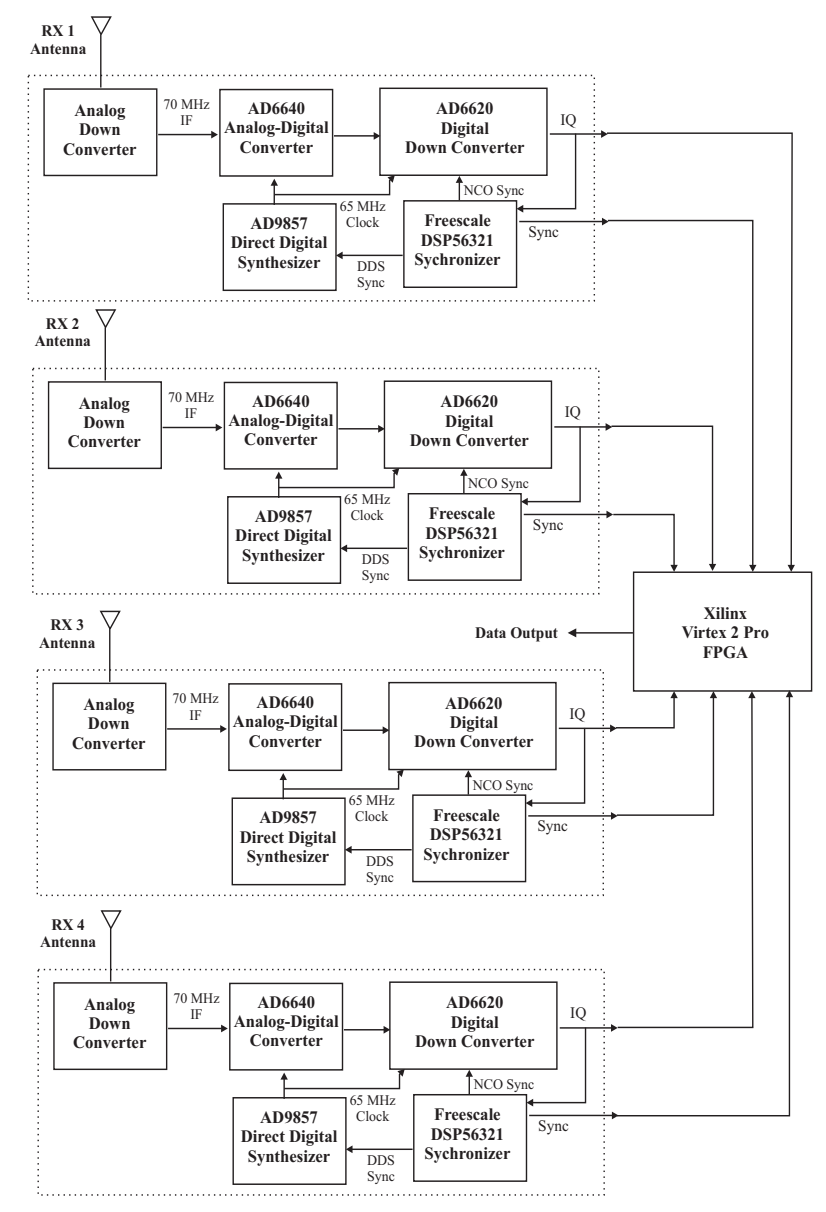

\section{Figure 1. SASRAT 4 receiver system architec- ture with Xilinx space-time decoder}

One set of buffered IQ signals are fed back into a Freescale DSP56321 platform through Port A which performs synchronization and channel estimation algorithms. The software for the DSP56321 is written in assembly language for optimum high speed performance. The output of this algorithm can dynamically control the Numerical Control Oscillator (NCO) in the AD6620 down convertor via Port B of the DSP or the AD9857 DDS through Port C to achieve synchronization.

In any digital receiver design, the major issues that must be solved involve the adjustment of gain in the receiver structure to work within the dynamic range of the signal processing algorithms, the ability to synchronize to the carrier frequency and phase, the ability to synchronize the time at which to sample the received symbols and the type of filtering needed for best possible bit-error performance. This paper does not cover the issues of gain control.

Carrier synchronization is required as the transmitter and the local oscillator (LO)in the receiver are derived from independent clock sources and will thus have a frequency offset. Doppler shift in the channel will also affect the received carrier frequency. Frequency offset will cause carrier phase rotation and correct decisions cannot be made on the rotating symbols. The local oscillator must be adjusted to stop this carrier rotation and continously track and correct any frequency offset that will occur over time.

We use a decoupled approach to carrier and timing synchronization. This allows the receiver to correct for carrier frequency offsets even when the symbol timing is not known. Wherever possible, we adopt a feedforward approach through the use of known training symbols (dataaided) or preambles to resolve phase ambiquity. This will also work in Rayleigh flat fading channels.

In our implementation to assist the receiver in correcting the frequency offset, a sync preamble is sent by the transmitter before data is sent. At the receiver, the I and Q data is processed by an algorithm depicted in Figure 2 which will adjust the frequency of the NCO to negate the frequency offset. On initialization and after a Data_Valid signal ap-

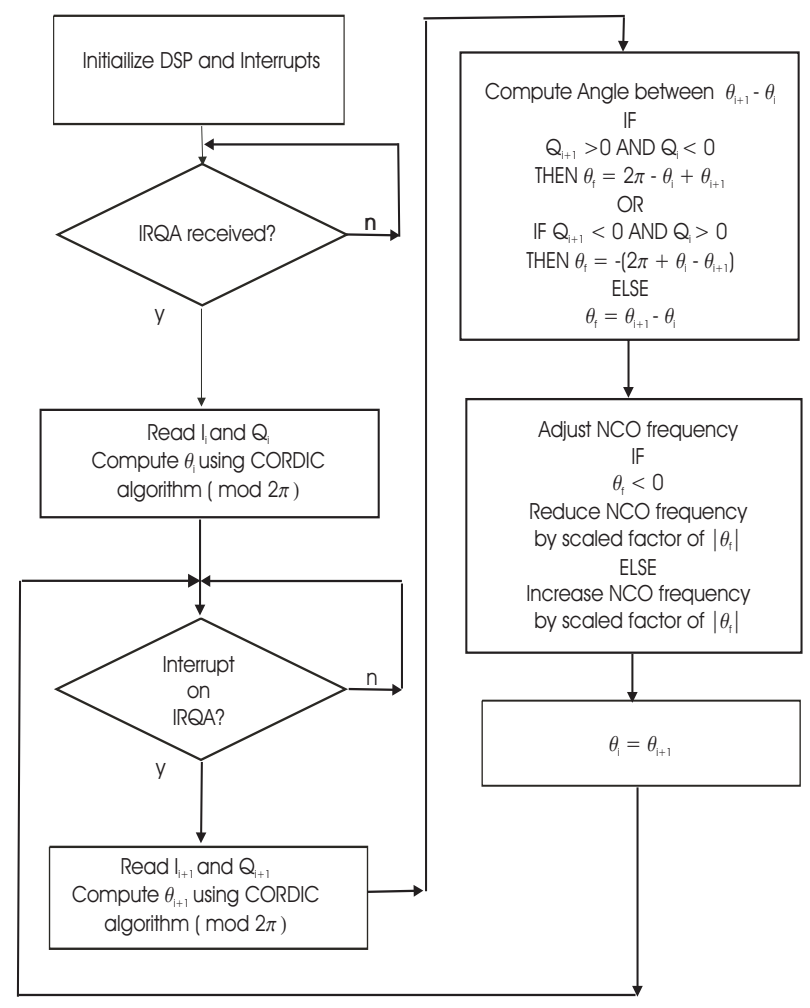

\section{Figure 2. Algorithm for Carrier Frequency Off- set Compensation}

pears on the Interrupt Request (IRQ) line of the DSP, the DSP reads the I and Q data stored in the 32-bit buffer. The 
algorithm then computes the phase angle of the vector using the Coordinate Rotation Digital Computer (CORDIC) algorithm [2]. The CORDIC algorithm is an iterative shift-add algorithm which can perform rectangular to polar conversions in DSPs' quickly and efficiently. This value is stored and the next IQ pair is then read and the new phase angle computed. The current and previous phases are compared and taking into account modulo $2 \pi$ operation, the difference is computed. As the timing period between the current and previous samples is known, the frequency offset can be estimated. A positive estimate indicates that the NCO frequency is below the TX carrier and the converse is true. Knowing the direction and speed of the rotating vector, the $\mathrm{NCO}$ is then programmed to correct for this. A new IQ pair is read and the process is repeated over the duration of the sync preamble.

A symbol sync preamble follows the carrier sync preamble. The symbol sync preamble allows the receiver to estimate and track the time at which to sample the received waveform. Symbol timing recovery will be implemented on the DSP56321 based on an all digital maximum likelihood symbol timing recovery algorithm by Watkins and Taylor [9]. The Watkins timing error detector (TED) works at baud rate and has performance comparable to the fractionally sampled $(2 \times$ baud rate) Gardner TED or the Amplitude Directed TED by Verdin and Tozer [3]. The Watkins TED also works well in Rayleigh flat fading channel.

In our complementary SASRATS transmitter platform [7] running a 2 transmit Alamouti encoding scheme, two symbols, $s_{0}$ and $s_{1}$ are transmitted simultanously from two transmitters at time instant $t$. At time instant $t+T$, the symbols $-s_{1}^{*}$ and $s_{0}^{*}$ are transmitted simultanously from the transmitters where * represents the complex conjugate. This is depicted in Figure 3. The transmitted symbols travel through 2 independent channels $h_{0}$ and $h_{1}$ to a receiver where noise and interference $n_{0}$ and $n_{1}$ are added to the received signal. $h_{0}$ and $h_{1}$ are complex multiplicative distortions assumed constant across two consecutive symbols. It is shown in [1] that at the input of the combiner, the receive signals are given by $r_{0}=r(t)=h_{0} s_{0}+h_{1} s_{1}+n_{0}$ and $r_{1}=r(t+T)=-h_{0} s_{1}^{*}+h_{1} s_{0}^{*}+n_{1}$. A channel estimator algorithm estimates $h_{0}$ and $h_{1}$ and this information is also fed to the combiner to yield two combined output signals $\tilde{s}_{0}=\left(\alpha_{0}^{2}+\alpha_{1}^{2}\right) s_{0}+h_{0}^{*} n_{0}+h_{1} n_{1}^{*}$ and $\tilde{s}_{1}=\left(\alpha_{0}^{2}+\alpha_{1}^{2}\right) s_{1}-h_{0} n_{1}^{*}+h_{1}^{*} n_{0}$ where $\alpha_{0}$ and $\alpha_{1}$ are the magnitudes of $h_{0}$ and $h_{1}$ respectively. The signals $\tilde{s}_{0}$ and $\tilde{s}_{1}$ together with the channel state information $h_{0}$ and $h_{1}$ are sent to the maximum likelihood (ML) detector so that ML estimates $\hat{s}_{0}$ and $\hat{s}_{1}$ can be made of $s_{0}$ and $s_{1}$. As we use PSK modulation of the symbols at the transmitter, the decision rule in the ML detector is to choose $s_{i}$ iff $d^{2}\left(\tilde{s}_{0}, s_{i}\right) \leq d^{2}\left(\tilde{s}_{0}, s_{k}\right), \forall i \neq k$ for $\hat{s}_{0}$ and choose $s_{i}$ iff $d^{2}\left(\tilde{s}_{1}, s_{i}\right) \leq d^{2}\left(\tilde{s}_{1}, s_{k}\right), \forall i \neq k$ for $\hat{s}_{1}$ where $d^{2}(x, y)$ is the

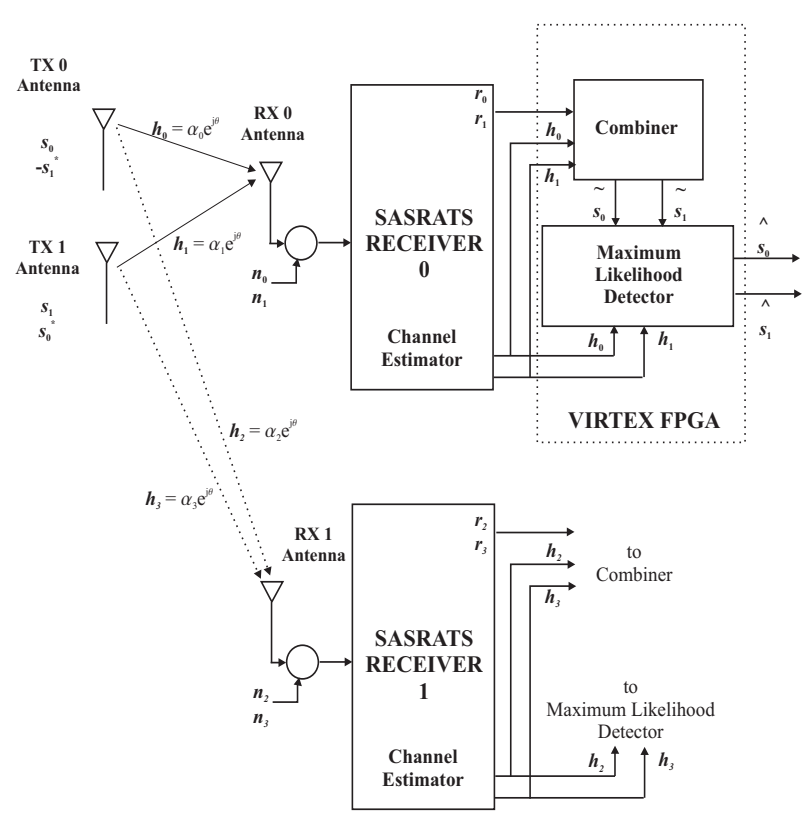

Figure 3. Block diagram of Alamouti decod-
ing implementation on SASRATS platform

squared Euclidean distance between signals $x$ and $y$.

The Alamouti scheme can be extended to more than one receiver but to implement a 2 transmitter and 2 receiver system for example, requires the estimation of 4 channels $\left(h_{0}, h_{1}, h_{2}\right.$ and $\left.h_{3}\right), 2$ at each receiver as shown in Figure 3. The decoding of the Alamouti encoded signals is a linear process and our SASRATS receiver system design will implement the combiner and maximum likelihood detection on the Xilinx Virtex 2 Pro FPGA board using the Xilinx Integrated System Enviroment (ISE) Foundation design tool. The channel estimation is carried out on the DSP platforms.

The channel estimation algorithm will be based on a feedforward data-aided (DA) technique based on a set of training symbols sent after the carrier and timing sync preambles where optimal minimum mean square error (MMSE) from the received training symbols can be performed via ML estimation. Data aided reception is of relatively low complexity since channel estimation (carrier synchronization) and detection are totally decoupled [8]. Each transmitter will have orthogonal preambles to enable the receiver to estimate the different channels $h_{0}$ and $h_{1}$ needed in the 2 TX 1 RX Alamouti decoding scheme mentioned above.

The hardware architecture allows high speed IQ and synchronization signals from up to 4 receivers to be combined into the FPGA board for processing. For more sophiscated space-time decoding experiments that require a Viterbi decoder or other decoding algorithms, the Xilinx Core Gener- 
ator modules can be used. The Xilinx Core Generator Intellectual Property (IP) modules [10] include math functions, correlators, filters, modulators, memory and storage elements incorporated into the ISE Foundation software. However, the Viterbi algorithm require an additional license.

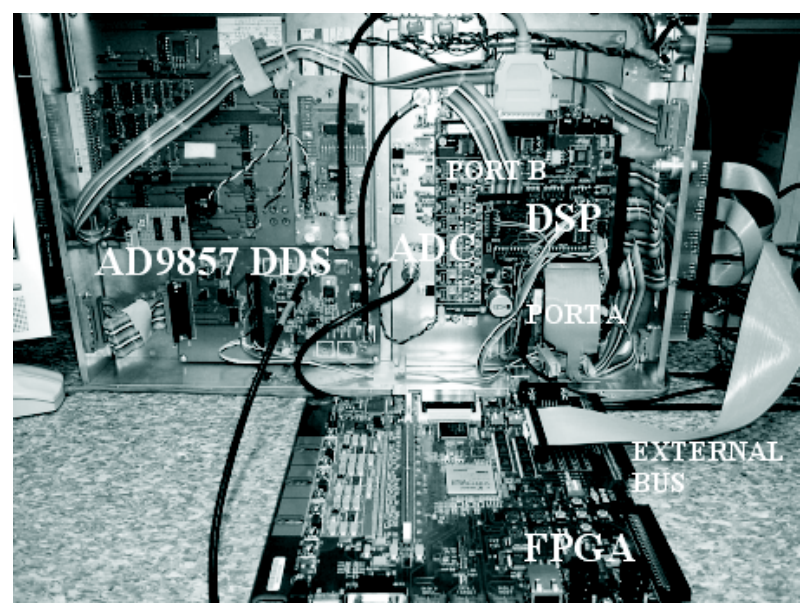

Figure 4. Photo of one SASRATS receiver with external FPGA board

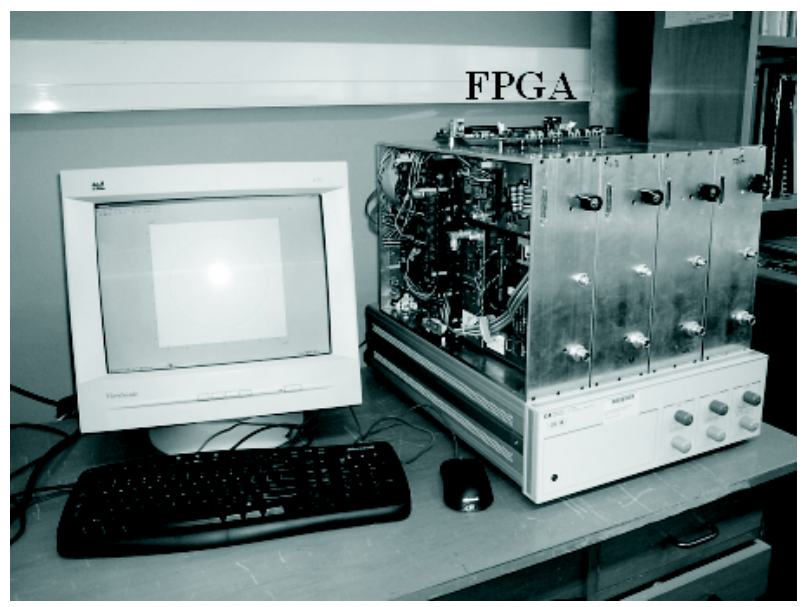

Figure 5. Photo of 4 SASRATS receivers with external FPGA board on top

Figure 4 shows the internal digital hardware of one receiver and Figure 5 shows the complete system consisting of 4 receivers. They are fully operational up to 5 Mbauds and fully software defined.

\section{Conclusions}

We have described the design and development of a real time software defined 4-receiver system and an Alamouti decoder to be implemented on an FPGA. Synchronization and decoder algorithms are fully defined with DSP software and FPGA firmware currently undergoing development and testing.

\section{References}

[1] S. Alamouti. Space block coding: A simple transmitter diversity technique for wireless communications. IEEE J. Select. Areas. Communication, 16:1451-1458, Oct. 1998.

[2] R. Andraka. A survey of CORDIC algorithms for FPGA based computers. Proc. ACM Int. Symp. on Field Programmable Gate Arrays, Monterey, CA, USA, pages 191200, 1998.

[3] R. Clark. On synchronization issues in wireless mobile digital communications. Master's thesis, University of Canterbury, 2002.

[4] D. Gesbert et al. From theory to practice: An overview of mimo space-time coded wireless systems. IEEE Journal on Selected Areas in Communications, 21:281-302, Apr. 2003.

[5] P. Green and D. Taylor. Smart antenna software radio test system. Proceedings of the First IEEE International Workshop on Electronic Design, Test and Applications., 1:68-72, Jan. 2002.

[6] P. Green and D. Taylor. Experimental verification of spacetime algorithms using the smart antenna software radio test system (sasrats) platform. Personal, Indoor and Mobile Radio Communications, 2004. PIMRC 2004. 15th IEEE International Symposium on, 4:2539-2544, 2004.

[7] P. Green and D. Taylor. Implementation of a high speed four transmitter space-time encoder using field programmable gate array and parallel digital signal processors. Proceedings of the Third IEEE International Workshop on Electronic Design, Test and Applications., Jan. 2006.

[8] M. M. H. Meyr and S. A. Fechtel. Digital Communication Receivers. Wiley, 1998.

[9] D. Taylor and G. Watkins. Ml symbol timing recovery in the rayleigh flat-fading channel using baud rate sampling. Conf. Proceedings of the 2001 International Symposium on Signals, Systems, and Electronics (ISSE'01), pages 62-65, 2001.

[10] Xilinx. http://www.xilinx.com/ ipcenter/ coregen/ coregen_iplists.htm, Nov. 2005. 